\title{
Electroencephalography source localization analysis in epileptic children during a visual working-memory task
}

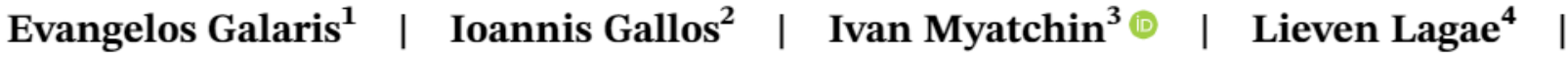 \\ Constantinos Siettos ${ }^{1}$
}

\begin{abstract}
${ }^{1}$ Dipartimento di Matematica e Applicazioni "Renato Caccioppoli", Universita' degli Studi di Napoli Federico II, Napoli, Italy

${ }^{2}$ School of Applied Mathematical and Physical Sciences, National Technical University of Athens, Athens, Greece

${ }^{3}$ Department of Anesthesiology, SintTrudo Regional Hospital, Sint-Truiden, Belgium

${ }^{4}$ Department of Development and Regeneration, Section Paediatric Neurology, KU Leuven, Leuven, Belgium
\end{abstract}

\section{Correspondence}

Constantinos Siettos, Dipartimento di Matematica e Applicazioni "Renato Caccioppoli”, Universita' degli Studi di Napoli Federico II, Napoli, Italy.

Email: constantinos.siettos@unina.it

\section{Funding information}

National Technical University of Athens; University of Naples Federico II

\begin{abstract}
We localize the sources of brain activity of children with epilepsy based on electroencephalograph (EEG) recordings acquired during a visual discrimination working memory task. For the numerical solution of the inverse problem, with the aid of age-specific MRI scans processed from a publicly available database, we use and compare three regularization numerical methods, namely the standardized low resolution brain electromagnetic tomography (sLORETA), the weighted minimum norm estimation (wMNE) and the dynamic statistical parametric mapping (dSPM). We show that all three methods provide the same spatio-temporal patterns of differences between the groups of epileptic and control children. In particular, our analysis reveals statistically significant differences between the two groups in regions of the parietal cortex indicating that these may serve as "biomarkers" for diagnostic purposes and ultimately localized treatment.
\end{abstract}

\section{KEYWORDS}

children, epilepsy, neuroimaging, numerical analysis, source localization

\section{1 | INTRODUCTION}

Epilepsy affects more than 65 million people worldwide while, approximately 1 out of 150 children is diagnosed with epilepsy during the first 10 years of their life. ${ }^{1}$ Although many children self-heal before adulthood, it has been shown that children with epilepsy confront various cognitive and behavioral problems such as problems in learning, attention and memory capacity. ${ }^{2}$ Thus, the systematic study of the brain (dys)functionalities of children with epilepsy, and ultimately the development of efficient/targeted treatments is one of the most challenging problems in neuroscience and beyond. Towards this aim, non-invasive neuroimaging techniques and in particular electroencephalograph (EEG) recordings are commonly used for clinical assessment. . $^{3-10}$

However, an analysis at the scalp level does not give insight to the malfunctioning of the actual brain regions and/or their connectivity. On the other hand, fMRI analysis can provide a better insight but it is limited by its low-time resolution. Thus, source localization, that is, the identification of the brain region activity from scalp/non-invasive recordings (usually EEG or MEG) has emerged a promising approach that can facilitate the analysis of brain activity as a clinical diagnostic tool. ${ }^{11,12}$ However, source localization is an 
ill-defined problem and as such, it poses open questions regarding its robustness and in general the validity of the obtained results. ${ }^{13}$

Comparative studies between the various numerical methods that aspire to solve the source localization problem are critical. ${ }^{14,15}$ Toward this aim, Jatoi et $\mathrm{al}^{16}$ have compared the standardized low resolution brain electromagnetic tomography (sLORETA) with the exact LORETA (eLORETA) based on EEG recordings of a visual experiment on healthy subjects. Cincotti et $\mathrm{al}^{17}$ compared two techniques for source localization, namely the surface Laplacian and LORETA using EEG recordings from a group of Alzheimer disease patients and age-matched controls. Yao and Devald ${ }^{18}$ compared the performances of several source localization methods on the basis of both synthetic and experimental EEG data of somatosensory evoked potentials. Attal and Schwartz ${ }^{19}$ compared the performance of three methods, namely the weighted minimum norm estimation (wMNE), sLORETA and the dynamic statistical parametric mapping (dSPM) for the characterization of distortions in cortical and subcortical regions using a realistic anatomical and electrophysiological model of deep brain activity. Seeland et $\mathrm{al}^{20} \mathrm{com}^{2}$ pared wMNE, sLORETA and dSPM using EEG data taken from eight subjects performing voluntary arm movements.

Regarding epilepsy, the majority of the studies have performed source localization with the aid of EEG-fMRI recordings and/or synthetic data approximating epileptic spatio-temporal patterns such as spikes and discharges. For example, Ioannides et $\mathrm{al}^{21}$ assessed the performance of two source localization methods, wMNE and eLORETA using MEG signals of ictal and interictal epileptiform discharges in epilepsy and K-complexes. Chowdhury et al $^{22}$ compared the performance of the coherent maximum entropy on the mean (cMEM) and the fourth order extended source multiple signal classification (4-ExSo-MUSIC) using MEG and EEG synthetic signals mimicking normal background and epileptic discharges. Hasan et $\mathrm{l}^{23}$ evaluated four algorithms (dSPM, wMNE, sLORETA and cMEM) using simulated data from a combined biophysical/physiological model used to generate interictal epileptic spikes as well as real EEG data recorded from one epileptic patient who underwent a full presurgical evaluation for drugresistant focal epilepsy. Moeller et $\mathrm{al}^{15}$ provide a review of the studies that used EEG-fMRI recordings to assess different types of epileptic form activity, underpinning the necessity for comparing with other methods including EEG source analysis.

Fewer studies have dealt with source-level analysis and compared different source localization methods using EEG clinical data taken by children with epilepsy. Among these studies, Adebimpe et $\mathrm{al}^{24}$ performed source localization using eLORETA to investigate changes in functional connectivity in 11 children with benign rolandic epilepsy (BRE) with centrotemporal spikes using resting-state EEG recordings using 12 subjects in the same age range as controls. Groening et $\mathrm{al}^{25}$ combined EEG-fMRI and EEG source analysis to identify epileptogenic foci in six children. Elshoff et $\mathrm{al}^{26}$ examined the efficiency of EEG-fMRI and EEG source analysis to localize the point of seizure onset in nine children with refractory focal epilepsy.

The above studies have focused mainly on the study of brain regions that are activated during seizure periods or before their onset. Several other studies have also aimed at analyzing the emerged patterns during seizure periods. For example, Fergus et $\mathrm{al}^{27}$ used a supervised machine learning approach to classify seizure and non-seizure records using an open dataset of seizured EEG signals from both children and adults.

On the other hand, it has been shown that studying epileptic seizure-free EEG recordings is of great importance as such analysis can facilitate the identification of patients at risk of epilepsy and/or forecast forth-coming seizures (for a discussion and review of ictal and interictal activity and their analysis see for example Reference 10).

Here, we perform a source-localization analysis of the brain activity of well-controlled epileptic children (with BRE and idiopathic generalized epilepsy [GE]) during a visual working memory (WM) task ${ }^{7}$. WM is commonly viewed as a functional integration system with limited capacity that is able to store information within a short-term register and simultaneously manipulate it on-line. Thus, WM is one of the most important components of information processing and its dysfunction leads to various problems in several cognitive functions including mental arithmetic, ${ }^{28}$ reading, ${ }^{29,30}$ decision making ${ }^{31}$ and reasoning. ${ }^{32}$ Epilepsy affects a lot the WM functioning as it has been shown by many studies. $^{33-37}$

Here, for the solution of the inverse problem, we use three methods, namely, sLORETA, ${ }^{38} \mathrm{dSPM}^{39}$ and wMNE. ${ }^{40}$ A statistical comparative analysis between methods and groups (healthy children vs children with epilepsy) revealed the crucial role of the superior parietal lobule (SPL) and inferior parietal lobule (IPL) at WM. Our findings are in line with fMRI studies ${ }^{41-44}$ that have shown that SPL and IPL are being involved in WM processing and thus can serve as a "biomarker" for identifying, monitoring and assessing epilepsy in children. 


\section{2 | MATERIALS AND METHODS}

\section{1 | Experimental procedure}

For a detailed description of the procedure please refer to Ref. 7.

\subsection{1 | Subjects}

In the study group, 21 children with established childhood epilepsy (age 6-16 years old; mean of 11.43 years, $\mathrm{SD} \pm 2.3$, 10 boys) were enrolled ${ }^{7}$. These children were diagnosed with one out of two following epilepsy syndromes: BRE $(n=9)$ and idiopathic GE $(n=12$, including childhood absence epilepsy $(n=5)$ and GE with tonic-clonic seizures $(n=7))$. All children were admitted to the neurophysiology laboratory of the University Hospital of Leuven for a 24-hours videoEEG monitoring during which the ERP study was done. They had no anti-epileptic treatment $(\mathrm{n}=3)$ or were on standard anti-epileptic medication (monotherapy, $\mathrm{n}=15$, duotherapy, $\mathrm{n}=3$ ), with drug dosages always being within normal ranges. Patients on monotherapy received valproic acid $(n=7)$, carbamazepine $(n=4)$, lamotrigine $(n=3)$ or sulthiame $(n=1)$. Patients on duotherapy received different drug combinations. ${ }^{7}$ None of the patients had structural brain abnormalities; in 18 patients, brain MRI was performed showing normal findings in all cases. Only patients with at least an 8 days seizure-free period preceding the test were included. ${ }^{7}$ Thereby we could avoid an acute effect of epileptic seizures on the child's performance. All children followed mainstream school and none had a history of learning problems.

As a control group, 25 age-matched non-epileptic children (mean age 10.76 years, $\mathrm{SD} \pm 3.4,17$ boys) were selected, who did not have any school problem either. The study protocol was approved by the Ethical Committee of University Hospital of Leuven. For more details about the experimental procedure please see Myatchin et al. ${ }^{7}$

\subsection{2 | Design and stimuli}

The event-related potentials study was done as part of video-EEG monitoring ${ }^{7}$. A visual one-backmatching working memory task was performed: children observed a continuous stream of seven different figures presented one after the other in pseudorandom order at the middle of a computer monitor, which was located at a distance of $1.0 \mathrm{~m}$ from the subject's eyes. Everyday figures were used (horse, wardrobe, jacket, cake, comb, bunch of grapes, hammer), white with a black contour on grey background, size $7.5 \mathrm{~cm} \times 6.5 \mathrm{~cm}$, visual angle $4^{\circ} 18^{\prime} \times 3^{\circ} 02^{\prime}$. Each stimulus was presented for 1.5 seconds, followed by a delay of 1.0 second, after which the next stimulus was presented. During the delay period a fixation point (dark-grey cross) was shown at the middle of the screen to facilitate eyes fixation. Any figure identical to the one immediately preceding it was defined as a target stimulus (probability .30). Children were asked to respond to all targets by pressing a button with their dominant hand. Both accuracy and speed were stressed. The single experimental block contained 120 trials, 36 of which were targets. The duration of the block was 5 minutes. This is an easy working memory task, which was chosen to ensure a good level of participant's performance.

First, the electrode placement and impedance calibration was performed. After that, the experimental procedure was described to the child. The child was seated comfortably in a dimly lit registration room and was instructed to look at the middle of the computer screen placed in front of him to avoid unnecessary eye movements; a fixation point (dark-grey cross) was shown between figures to facilitate eye fixation. The child was also instructed to avoid movements to reduce muscle artifacts in the EEG signal. The instruction for the task was given directly before the task. During the experiment, no interaction with the experimenter was allowed during the task and the experimenter sat out of sight of the child.

\subsection{3 | EEG recordings}

Nineteen $\mathrm{Ag} / \mathrm{AgCl}$ electrodes (Technomed Europe) were placed according to the international 10 to 20 system at Fp1, Fp2, F3, F4, F7, F8, Fz, C3, C4, Cz, T3, T4, T5, T6, P3, P4, Pz, O1 and O2 ${ }^{7}$. The placement of additional four EOG electrodes resulted in two EOG channels: horizontal EOG—-two electrodes on the outer canthi of eyes, and vertical EOG- 
two electrodes above and below one eye. EOG channels allowed us to detect both vertical and horizontal eye movements in order to effectively remove them from EEG recording during subsequent preprocessing of the signal (see below). Two linked mastoid electrodes were used as a reference. EEG was sampled at a frequency of $1000 \mathrm{~Hz}$ with 12 bits A/D converter and amplified using a band-pass filter of $0.095 \mathrm{~Hz}$ to $70 \mathrm{~Hz}$. Notch filter was off. Registration of the digital EEG was made using the software program BrainlaB 4.0 (OSG, Belgium). The impedance of all electrodes was monitored for each subject prior to recording and was always kept below $5 \mathrm{k} \Omega$.

\subsection{4 | Data pre-processing}

Data pre-processing was performed offline using the EEGLAB v.5.02 toolbox (Matlab 7.0.4 platform).$^{45}$ The ECG channel was factored out. Data were filtered with a $50 \mathrm{~Hz}$ digital low pass filter. Eye movement artifacts were marked and removed from the continuous signal without affecting the signal itself using an ICA-based algorithm. ${ }^{7}$ EEG fragments containing movement artifacts as well as any epileptic activity were removed based on visual inspection of the data. This resulted in an EEG signal clean from (eye) movement artifacts and epileptic activity, which was then used for further analysis. Afterwards, the continuous EEG signal was epoched according to the type of stimulus (target and non-target), with $200 \mathrm{~ms}$ pre-stimulus (delay period) and $400 \mathrm{~ms}$ poststimulus (presentation period of the second stimulus, where the motor responses had not yet taken place). Omitted target trials (ie, trials without correct motor response) and committed non-target trials (ie, trials with a wrong motor response) were excluded from the analysis. We then performed a down-sampling at $500 \mathrm{~Hz}$ and we applied a baseline correction by subtracting the mean value of the $200 \mathrm{~ms}$ of the pre-stimulus period. Overall, we ended up with 92 datasets ( 21 epileptic $\times 2$ trial types +25 control $\times 2$ trial types) of 19 multi time series, which were divided into four group types (epileptic-target [ET], epileptic-non-target [ENT], control target [CT], control non-target [CNT]); a further subdivision into benign rolandic target (BRE)T, idiopathic generalized epilepsy target (GE)T and benign rolandic non-target (BRE)NT, idiopathic generalized epilepsy non-target (GE)NT was also considered for investigating any differences between the two epileptic types.

\section{2 | Numerical solution of the source localization problem}

Source localization aims at identifying the (unknown) sources of the brain from data taken usually from noninvasive electromagnetic recording (here: EEG recordings). Its solution involves a forward and an inverse problem. The forward problem refers to the calculation of the electric potentials of the electrodes starting from a given electrical source. The solution of the forward problem is related to the construction of a head model. The head model contains both anatomical information and the conductivities of three layers, namely the skull, the cortex and the scalp. ${ }^{46}$ Anatomical images can be obtained experimentally with the aid of MRI scans, while volume conduction models can be constructed using, for example, the $\mathrm{BEM}^{47}$ or the FEM. ${ }^{48}$ The head model volume is tessellated into small-sized cubes, the voxels. Sources may be associated to single voxels or clusters of voxels. Here, each voxel is associated to a single source. The relation between the scalp recordings and the discretized head model volume is performed using the linear matrix equation:

$$
V=G x+\epsilon,
$$

where $V$ is a known $N \times 1$ matrix which contains the time instances as recorded by each channel ( $N$ is the number of channels), $x$ is the unknown $M \times 1$ matrix of the intensities of the $M$ sources ( $M$ is the number of voxels).

The matrix $G$, with dimensions $N \times M$ is the so-called lead field matrix that contains the information of the head geometry and conductivities. $G$ is known (from the solution of the so-called forward problem [see eg, in Reference 49]) and is related with the head model $^{50} ; \epsilon$ reflects the noise in the measurements.

The inverse problem is ill-defined, as there is an infinite number of combinations of positions and intensities that could effectively produce the electric potentials and magnetic fields measured. The general idea behind its solution is to express it as a linear optimization problem with regularization: 


$$
\hat{x}=\min _{x}\left(\|V-G x\|_{2}^{2}+\sum_{i=1}^{k} a_{i}\left\|W_{i} x\right\|_{p}\right) .
$$

In the above equation, $k$ is the number of regularization constraints (reflecting the a-priori physiological information) (usually k=1); the matrix $W$, of dimension $M \times M$ is a weighted matrix related to the imposed constraints; $\alpha_{i}$ is the regularization parameter and denotes the importance of every constraint.

For different choices of $W, k$ and $p$ (reflecting the type of the norm), we get different methods.

Here, for our analysis, we used and compared three differen $t^{20}$ methods, namely the wMNE, the sLORETA and the dSPM that are described below.

\subsection{1 | Weighted minimum norm estimation}

For $W=I$ (the identity matrix) and $P=2$ (the $L-2$ norm) in Equation (2) we get the minimum norm estimation (MNE). ${ }^{51} \mathrm{MNE}$ uses the mathematical assumption that the best solution, through the infinite set of solutions, is the one with the minimum norm. Despite the fact that MNE was the first method used to extract a 3D distributed solution, the simplicity of its assumption often leads to inadequate solutions. In particular, it has been shown, that this method fails in identifying deep sources. ${ }^{52}$ Because of the minimum norm constraint, sources that are located in deep regions are moved closer to the cortex.

The wMNE method is a variation of the MNE that improves the problem of the mislocation of the deep sources. wMNE uses instead of the identity matrix, a diagonal matrix $W_{c}$ of dimension MxM, that contains the weighting factors. From the multiple choices that can be chosen as weighted factors, usually chosen as $W_{c}=\operatorname{diag}\left(\left\|G_{i}\right\|_{2}\right)$ (for $\left.i=1, \ldots, M\right)$ (i.e. based on the norm of the columns of the matrix $\mathrm{G}$ ). ${ }^{53}$ Then, the unique solution is given by:

$$
x_{\mathrm{wMNE}}=L V,
$$

where $L=\left(W_{c}^{T} W_{c}\right)^{-1} G^{T}\left(G\left(W_{c}^{T} W_{c}\right)^{-1} G^{T}+a C_{\varepsilon}\right)^{-1}$ is called the inverse operator with dimensions $(M \times N)$.

\subsection{2 | The dynamic statistical parametric mapping}

The $\mathrm{dSPM}^{39}$ is similar to the wMNE but uses a different regularization. dSPM computes the source estimates of the noise based on the noise covariance matrix $C_{e}=\alpha H$ and normalizes the rows of the inverse operator.

$H=I_{N x N}-\left(11^{\mathrm{T}} / 1^{T} 1\right)$ is the NxN centering matrix and plays the role of the identity matrix in the measurement space; $1 \in \mathrm{R}^{\mathrm{Nx} 1}$ is a vector of ones Then, from Equation (3), the source estimates of the noise form a diagonal matrix:

$$
C_{\hat{x}}=L C_{\varepsilon} L^{T}
$$

Thus, the dSPM solution is given by:

$$
x_{\mathrm{dSPM}}=L_{\mathrm{dSPM}} V \text {, }
$$

where $L_{\mathrm{dSPM}}=W_{\mathrm{dSPM}} L$, where $W_{d S P M}$ is a diagonal matrix with elements derived by $C_{\hat{x}}$

\subsection{3 | Standardized low resolution brain electromagnetic tomography}

sLORETA considers another source of variance, except from the covariance of the measurement noise $C_{\varepsilon}$, the covariance of the actual sources $C_{x}=I_{N x N}$. Assuming that the activity of the actual sources and the noise of the measurements are uncorrelated and based on the linear relation of Equation (1), we have $\mathrm{e}^{38}$ : 


$$
C_{V}=G C_{x} G^{T}+C_{\epsilon}=G G^{T}+\alpha H .
$$

Substituting Equation (6) in (3), setting $W_{c}=I_{M x M}$, we can estimate the variation of the estimated sources as:

$$
C_{\hat{x}}=L C_{V} L^{T}=L\left(G G^{T}+\alpha H\right) L^{T}=G^{T}\left(G G^{T}+\alpha H\right)^{-1} G .
$$

The covariance of the estimated sources is equivalent to the Backus and Gilbert resolution matrix, ${ }^{54}$ which is given by plugging Equation (1) into (3) and substituting the inverse operator to get $^{38}$ :

$$
x_{\text {SLORETA }}=G^{T}\left(G G^{T}+a H\right)^{-1} V=A x=C_{\hat{x}} x,
$$

where $A=L G$ is the resolution matrix.

\subsection{4 | Head models for children}

In our study, we did not have individual MRI scans for each child that participated to the experiment. Thus, in the absence of such specific information, we used age-specific MRI templates for children acquired from the "Neurodevelopmental MRI database". ${ }^{55-58}$ The goal of this database is to provide for research purposes, exactly in the absence of specific MRI scans, a series of age-appropriate average MRI reference templates and related information. Each template was constructed using identical procedures to facilitate comparisons across lifespan. The database consists of average templates (T1W and T2W), segmenting priors, and stereotaxic atlases. ${ }^{55}$ The "Neurodevelopmental MRI Database" is available online (http://jerlab.psych.sc.edu/NeurodevelopmentalMRIDatabase/). The database is publicly available to researchers upon request for clinical and experimental studies of normal and pathological brain development. The data is shared under a Creative Commons Attribution-NonCommercial-Noderivs 3.0 Unported License (CC BY-NC-ND 3.0; http://creativecommons.org/licenses/by-nc-nd/3.0/deed.en\$_\$US).

Using this database, we were able to construct an "average" age-specific head model for each child taking into account its age. For our study, we constructed 11 averaged head models (taking into account the database with head models of children between 6 and 16 years old, that is, one "average" head model per year). In Table 1, we provide information about the total number of MRI scans per age.

Here, for the construction of the head models, as skull conductivities are age-dependent, ${ }^{59}$ we used different conductivities ratios (CR, cortex/skull) for every age-dependent model. The conductivity value for scalp and cortex was set to the standard value of $0.33 \mathrm{~S} / \mathrm{m} .{ }^{60}$ Table 2 presents analytically the different conductivity ratios for every age. ${ }^{60}$

\begin{tabular}{llllllllllll} 
Age & 6 & 7 & 8 & 9 & 10 & 11 & 12 & 13 & 14 & 15 & 16 \\
$1.5 \mathrm{~T}$ & 27 & 27 & 46 & 46 & 62 & 31 & 37 & 34 & 32 & 32 & 34 \\
$3.0 \mathrm{~T}$ & 10 & & 19 & & 16 & & 15 & 11 & 30 & & 13 \\
Combined & 37 & 27 & 56 & 46 & 72 & 31 & 47 & 34 & 42 & 32 & 44 \\
\hline
\end{tabular}

T A B LE 1 Total number of scans per age for $1.5 \mathrm{~T}, 3.0 \mathrm{~T}$ and combined average MRI templates

Note: All 1.5 T MRIs and part of 3.0 T MRIs are included in the "Combined" column as in the original publications. ${ }^{55,57}$

\begin{tabular}{lllllll} 
Age & 6 & $7-8$ & $9-10$ & $11-12$ & $13-14$ & $15-16$ \\
CR & 15 & 20 & 30 & 40 & 50 & 60 \\
\hline
\end{tabular}

TABLE 2 Conductivity ratios (cortex/skull) for every age-dependent head model

Note: The standard conductivity value for scalp and cortex was set to $0.33 \mathrm{~S} / \mathrm{m} .{ }^{60}$ 


\subsection{Statistical analysis}

After the data pre-processing and the implementation of the source-localization algorithms, as described in the previous section, we get 92 time-series at the source space. Our data have a spatio-temporal structure: number of voxels (spatial dimension) and time points (time dimension).

Here, we sought for statistically significant differences between (a) the groups of BRE and idiopathic GE, (b) the groups of healthy controls (C) and epileptic children (E) in total, and (c) the three different source-localization methods. For the comparisons, we performed two-sample $t$ tests based on three basic assumptions. In particular, in order to perform the $t$ tests, we checked if (a) the amplitude of the source signal at each voxel and at each time instant follows a normal distribution among subjects in a group; (b) the variances of the amplitude of the source signal at each voxel and at each time instant of (BRE)T, (GE)T, (BRE)NT, (GE)NT, CT, ET, CNT, ENT are equal; and (c) the amplitudes of the source signal at each voxel and at each time instant are independent for (BRE)T, (GE)T, (BRE)NT, (GE)NT, CT, ET, CNT, ENT.

For the test of normality, we used the Shapiro-Wilk test. ${ }^{61}$ The null hypothesis of the test is that a sample comes from a normal distribution. The test statistic reads:

$$
W=\frac{\left(\sum_{i=1}^{M} \alpha_{i} x_{(i)}\right)^{2}}{\sum_{i=1}^{M}\left(x_{i}-\bar{x}\right)^{2}},
$$

where $x_{i}, i=1,2, \ldots M$ is the intensity of the source $i, x_{(i)}$ is the $i$-th order statistic, $\bar{x}$ is the sample mean and coefficients $\alpha_{i}$ are given by $\left(\alpha_{1}, \alpha_{2}, \ldots, \alpha_{M}\right)=\left(m^{T} C_{V}{ }^{-1} / C\right)$. $C$ is a vector norm: $C=\left\|C_{V}{ }^{-1} m\right\|, m=\left(m_{1}, m_{2}, \ldots, m_{M}\right)^{T}$ are the expected values of the order statistics of independent and identically distributed random variables sampled from the standard normal distribution and $C_{V}$ is the covariance matrix of those normal order statistics. $F$ tests were performed to validate the second assumption (ie, the equality of the variances of the amplitude values of each voxel between the different group comparisons.

For the comparison between the groups of epileptic syndromes, we considered the pairs (BRE)T vs (GE)T and (BRE)NT vs (GE)NT. For the comparison between the control and epileptic groups, we considered the pairs CT vs ET and ENT vs CNT. Thus, we tested for normality and equality of variances for each voxel and each time sample (ie, we have performed a total of $5.148 \times 600=3.088 .800 t$ tests). The level of significance was set to $P<.05$, meaning that the risk of taking a false positive is $5 \%$ of the cases. Here, in order to deal with the multicomparison problem, we used the FDR correction. ${ }^{62}$ The null hypothesis $H_{0}$ for all comparisons was that the two different groups have equal means regarding the emerged spatio-temporal activation at the source level. Based on the above, the $H_{0}$ hypothesis could not be rejected. Similarly, the $F$ tests validated also the second assumption. The independence is reasonably assumed to hold true. Having guaranteed that the $t$ test can be applied, we proceeded with the comparisons between the different groups and methods.

Thus, we performed $3 . E^{6}$ simultaneous two-sample $t$ tests with $P<.05$ level of significance. FDR was used to deal with the multicomparison problem. Another constraint that we added to avoid spurius and random effects was the one of the minimum duration of the activations. Thus, we excluded all the signals that were statistically significantly for time intervals less than $50 \mathrm{~ms}$.

In order to detect statistically significant differences between the three methods at the source level, we also performed paired $t$ tests taking the absolute difference between the signals at the source level. Again, in order to deal with the multicomparison problem the FDR correction was used. This processing was performed to detect possible different brain areas induced by the different inverse algorithms and not by the original brain activity. We excluded all the signals that were statistically significantly for time intervals less than $50 \mathrm{~ms}$ as before. Furthermore, we also applied a space constraint (of 30 voxels) in order to exclude sparse voxels (or small-size groups of voxels) that do not represent a coherent brain area.

\section{3 | RESULTS}

For our analysis, we used the BrainStorm toolbox for Matlab. ${ }^{63}$ The source-reconstructed time series were obtained by combining the EEG recordings with the appropriate (respect to the age of the subject) constructed MRI templates. From each template, we extracted three layers (scalp, inner skull, outer skull) and the source space (cortical surface). The 
number of vertices for each layer were set to 2562 vertices for each surface. Then, the volume conduction models were constructed in openMEEG software ${ }^{64}$ which uses the BEM. The space resolution for the source model was set to 5124 voxels with fixed orientation perpendicular to the cortex surface.

Thus, the time series at the source level were reconstructed using wMNE, dSPM and SLORETA. The noise was computed from the raw EEG data using the pre-stimulus period for baseline correction and then the noise covariance matrix was calculated. A parameter that has to be determined is the "signal to noise ratio" (SNR). In Brainstorm, the computation of SNR is performed as in the original MNE software of Hamalainen. ${ }^{65}$ The signal covariance matrix is "whitened" by the noise covariance matrix and the square root of the mean of its spectrum yields the average amplitude of SNR. The default value in Brainstorm is set to 3 .

For our illustrations, we have split the time period to three main intervals: the pre-stimulus period [ $-200 \mathrm{~ms} 0 \mathrm{~ms}$ ), the period exactly after the stimulus [0 ms-199 ms] and the post-stimulus period [ $200 \mathrm{~ms}-400 \mathrm{~ms}$ ).

Following the statistical analysis described in the methodology, the comparison between the epileptic group of BRE and that of GE revealed no significant differences at the source-level.

At the next step we considered the comparison between the group of control and the group of epileptic (BRE and GE). The main results of the source localization procedure for these groups are presented analytically in Table 3 .

In Table 4, we also provide the numerical residuals for each method (the L2-norm (res $=\|V-G \hat{x}\|_{2}$, where $G$ is the forward operator and $\hat{x}$ the estimated amplitudes), as well as the corresponding values of the regularization terms.

The statistical analysis between the groups (normal vs epileptic) revealed that all methods gave relatively similar results. The pair $t$ test between ENT and CNT revealed a statistically significant difference in the time-range of $170 \mathrm{~ms}$ to $230 \mathrm{~ms}$.

T A B LE 3 Group averaged sources as obtained by the three methods: wMNE, dSPM and sLORETA

\begin{tabular}{|c|c|c|c|c|c|}
\hline Time period & Method & CT & CNT & ET & ENT \\
\hline \multirow[t]{4}{*}{$\begin{array}{l}\text { Pro-stimulus (-200- } \\
\quad-1 \mathrm{~ms})\end{array}$} & wMNE & $\begin{array}{l}\text { Right occipital lobe } \\
\text { ( } \sim 140 \text { voxels })\end{array}$ & $\begin{array}{l}\text { Occipital lobe }(\sim 100 \\
\text { voxels })\end{array}$ & $\begin{array}{l}\text { Right occipital lobe } \\
\text { ( } \sim 90 \text { voxels })\end{array}$ & $\begin{array}{l}\text { Occipital lobe ( } \sim 80 \\
\text { voxels })\end{array}$ \\
\hline & dSPM & $\begin{array}{l}\text { Right occipital lobe } \\
\text { ( } \sim 220 \text { voxels })\end{array}$ & $\begin{array}{l}\text { Right occipital lobe } \\
\text { ( } \sim 150 \text { voxels })\end{array}$ & $\begin{array}{l}\text { Right occipital lobe } \\
\text { ( 160 voxels })\end{array}$ & $\begin{array}{l}\text { Occipital lobe }(\sim 190 \\
\text { voxels })\end{array}$ \\
\hline & sLORETA & $\begin{array}{l}\text { Right occipital lobe } \\
\quad(\sim 530 \text { voxels })\end{array}$ & $\begin{array}{l}\text { Occipital lobe }(\sim 230 \\
\text { voxels })\end{array}$ & $\begin{array}{l}\text { Right occipital lobe } \\
\quad(\sim 290 \text { voxels })\end{array}$ & $\begin{array}{l}\text { Right occipital lobe } \\
\text { ( } \sim 270 \text { voxels })\end{array}$ \\
\hline & & & $\begin{array}{l}\text { Left parietal lobe } \\
\qquad(\sim 150 \text { voxels })\end{array}$ & & \\
\hline \multirow[t]{6}{*}{$\begin{array}{l}\text { Exactly after stimulus } \\
\quad(0-199 \mathrm{~ms})\end{array}$} & wMNE & $\begin{array}{l}\text { Occipital lobe }(\sim 120 \\
\text { voxels })\end{array}$ & $\begin{array}{l}\text { Right occipital lobe } \\
\quad(\sim 100 \text { voxels })\end{array}$ & $\begin{array}{l}\text { Occipital lobe ( } \sim 90 \\
\text { voxels) }\end{array}$ & $\begin{array}{l}\text { Superior parietal lobe } \\
\quad(\sim 50 \text { voxels })\end{array}$ \\
\hline & & & & & $\begin{array}{l}\text { Occipital lobe }(\sim 110 \\
\text { voxels })\end{array}$ \\
\hline & dSPM & $\begin{array}{l}\text { Right occipital lobe } \\
\text { ( 330 voxels) }\end{array}$ & $\begin{array}{l}\text { Right occipital lobe } \\
\text { ( } \sim 310 \text { voxels })\end{array}$ & $\begin{array}{l}\text { Occipital lobe }(\sim 240 \\
\text { voxels })\end{array}$ & $\begin{array}{l}\text { Superior parietal lobe } \\
\quad(\sim 100 \text { voxels })\end{array}$ \\
\hline & & & & & $\begin{array}{l}\text { Right occipital lobe } \\
\text { ( } \sim 330 \text { voxels })\end{array}$ \\
\hline & sLORETA & $\begin{array}{l}\text { Right occipital lobe } \\
\quad(\sim 590 \text { voxels })\end{array}$ & $\begin{array}{l}\text { Occipital lobe }(\sim 510 \\
\text { voxels })\end{array}$ & $\begin{array}{l}\text { Occipital lobe }(\sim 450 \\
\text { voxels })\end{array}$ & $\begin{array}{l}\text { Superior parietal lobe } \\
\text { ( } \sim 240 \text { voxels })\end{array}$ \\
\hline & & & & & $\begin{array}{l}\text { Occipital lobe }(\sim 500 \\
\text { voxels })\end{array}$ \\
\hline \multirow[t]{3}{*}{$\begin{array}{l}\text { Post-stimulus } \\
\qquad(200-399 \mathrm{~ms})\end{array}$} & wMNE & $\begin{array}{l}\text { Parietal lobe }(\sim 70 \\
\text { voxels })\end{array}$ & $\begin{array}{l}\text { Right parietal lobe } \\
\text { ( } \sim 60 \text { voxels })\end{array}$ & $\begin{array}{l}\text { Right parietal lobe } \\
\text { ( } \sim 60 \text { voxels })\end{array}$ & $\begin{array}{l}\text { Right parietal lobe } \\
\text { ( } \sim 80 \text { voxels })\end{array}$ \\
\hline & dSPM & $\begin{array}{l}\text { Parietal lobe }(\sim 180 \\
\text { voxels })\end{array}$ & $\begin{array}{l}\text { Parietal lobe }(\sim 110 \\
\text { voxels })\end{array}$ & $\begin{array}{l}\text { Right parietal lobe } \\
\text { ( } \sim 200 \text { voxels) }\end{array}$ & $\begin{array}{l}\text { Parietal lobe ( } \sim 190 \\
\text { voxels })\end{array}$ \\
\hline & sLORETA & $\begin{array}{l}\text { Parietal lobe ( } \sim 390 \\
\text { voxels })\end{array}$ & $\begin{array}{l}\text { Parietal lobe ( } \sim 360 \\
\text { voxels })\end{array}$ & $\begin{array}{l}\text { Right parietal lobe } \\
\text { ( } \sim 470 \text { voxels })\end{array}$ & $\begin{array}{l}\text { Parietal lobe }(\sim 500 \\
\text { voxels })\end{array}$ \\
\hline
\end{tabular}


TABLE 4 Mean and SD values for the L2-norm residuals and the regularization term for each method and each group

\begin{tabular}{|llll|} 
Method & Group & Residuals $(\boldsymbol{\mu V})$ & Regularization term $(\mathbf{n A - m})$ \\
\hline wMNE & CT & $4.53 \pm 0.94$ & $13.2 \pm 2.14$ \\
& ET & $5.34 \pm 1.02$ & $15.41 \pm 3.23$ \\
\hline dSPM & CNT & $3.63 \pm 1.25$ & $13.13 \pm 3.42$ \\
& ENT & $6.42 \pm 1.76$ & $16.31 \pm 3.67$ \\
& CT & $3.92 \pm 0.71$ & $12.78 \pm 3.12$ \\
& ET & $5.21 \pm 1.18$ & $15.91 \pm 3.54$ \\
& CNT & $4.11 \pm 1.37$ & $14.34 \pm 4.15$ \\
& ENT & $7.08 \pm 1.58$ & $17.37 \pm 4.52$ \\
& CT & $3.12 \pm 0.59$ & $10.91 \pm 3.19$ \\
& ET & $4.04 \pm 0.86$ & $11.46 \pm 2.84$ \\
& CNT & $2.98 \pm 0.43$ & $11.51 \pm 2.9$ \\
& ENT & $3.45 \pm 0.73$ & $12.43 \pm 3.57$ \\
\hline
\end{tabular}

Abbreviations: CNT, control non-target; CT, control target; ENT, epileptic non-target; ET, epileptic target.

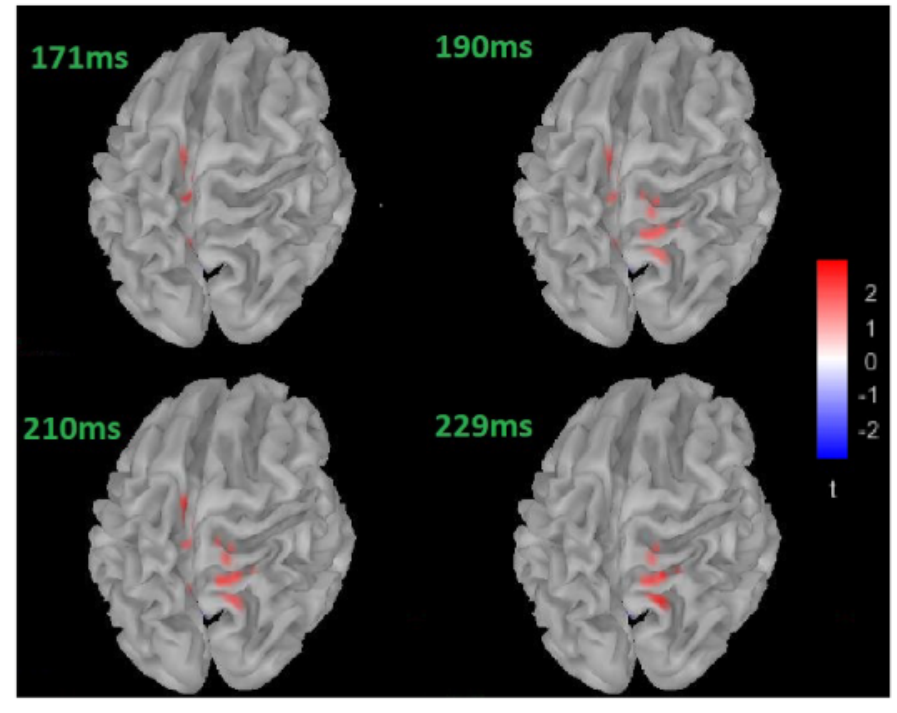

(A) wMNE

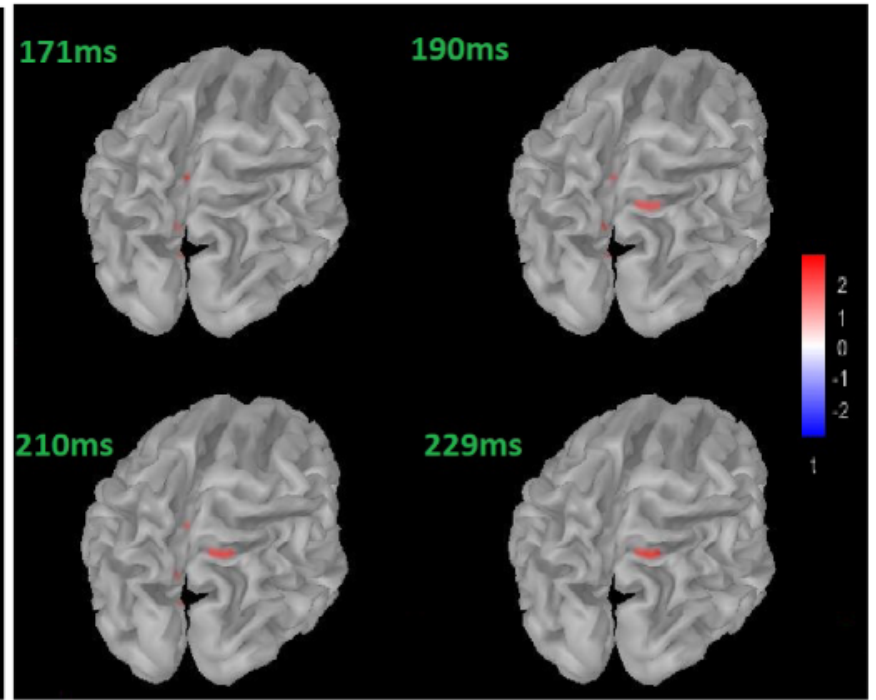

(B) dSPM

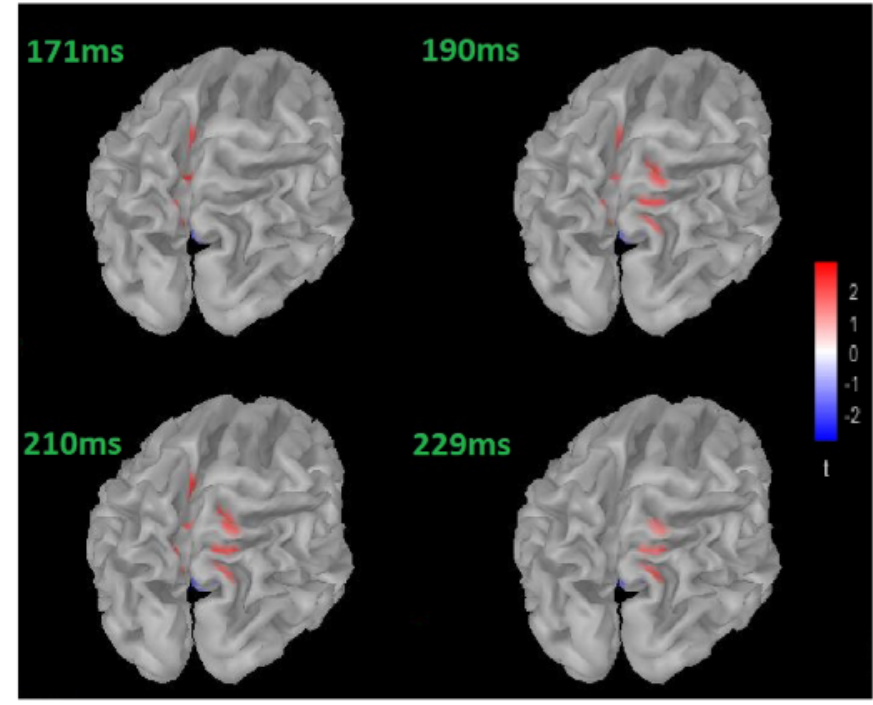

(C) SLORETA

FI G U RE 1 ENT vs CNT: The SPL ( superior parietal lobule) mainly of the right hemisphere is activated more for the ENT group at the time interval from $170 \mathrm{~ms}$ to $230 \mathrm{~ms}$. (A) wMNE, (B) dSPM, (C) sLORETA 


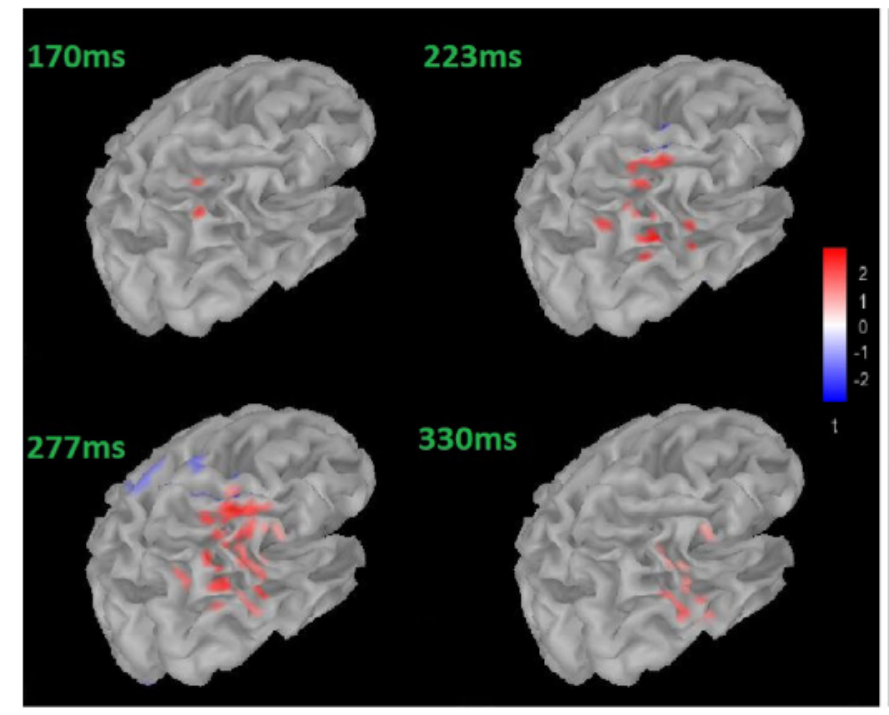

(A) WMNE

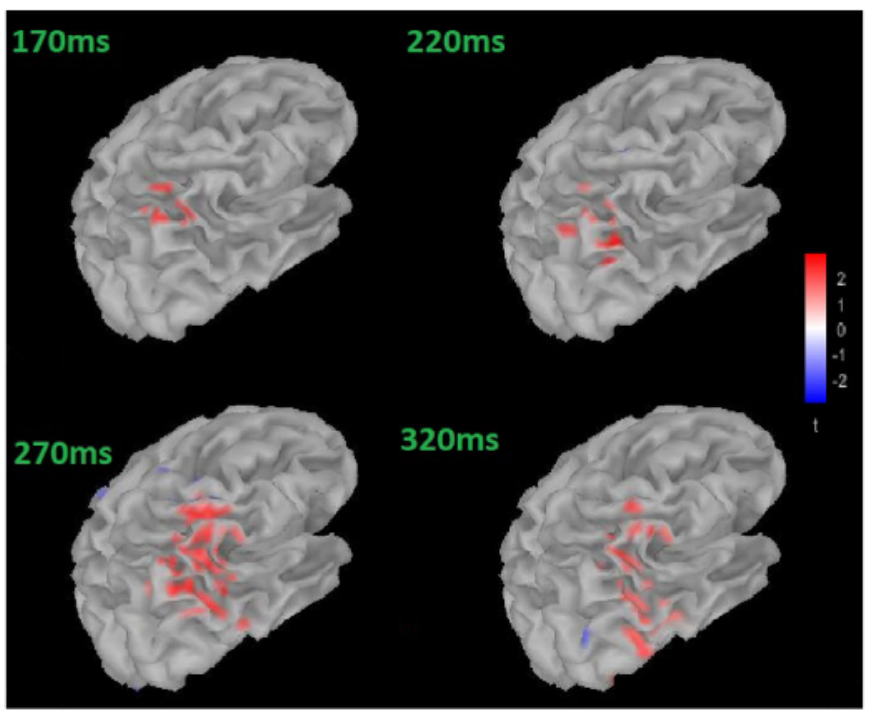

(B) dSPM

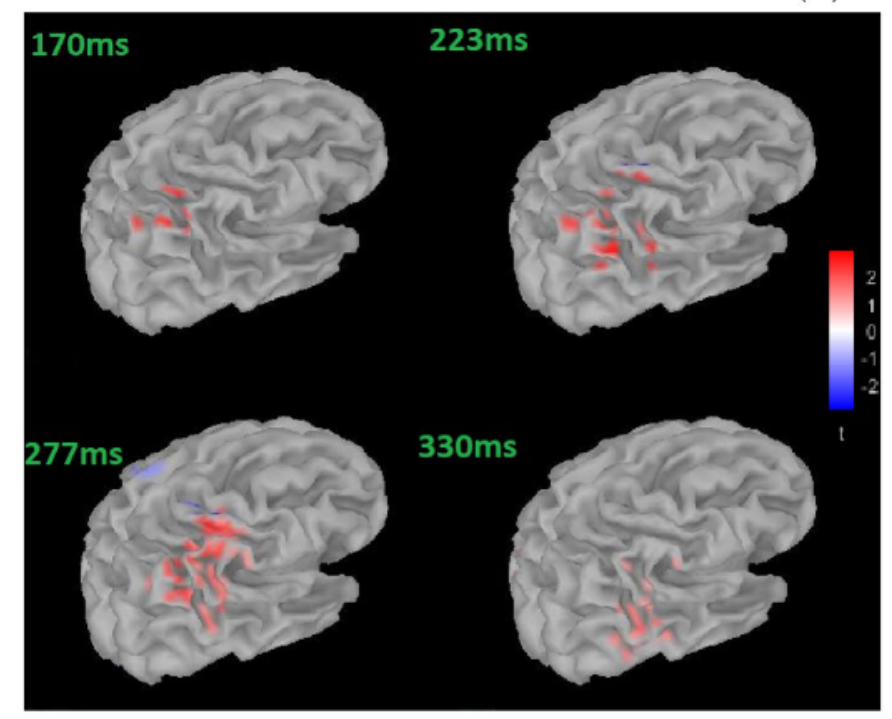

(C), SLORETA

FI G U RE 2 ET vs CT: The IPL (inferior parietal lobule) of the right hemisphere is activated more for the ET group at the time interval from $160 \mathrm{~ms}$ to $360 \mathrm{~ms}$. (A) wMNE, (B) dSPM, (C) sLORETA

T A B LE 5 Analytical presentation of the statistically significant differences for ET vs CT and ENT vs CNT

\begin{tabular}{|lllllll} 
Comparison & $\begin{array}{l}\text { Time } \\
\text { period }(\mathbf{m s})\end{array}$ & $\begin{array}{l}\text { Group } \\
\text { activated more }\end{array}$ & $\begin{array}{l}\text { Brain } \\
\text { region }\end{array}$ & $\begin{array}{l}\text { Brodmann } \\
\text { area }\end{array}$ & $\begin{array}{l}\text { Number of } \\
\text { voxels }\end{array}$ & Comments \\
\hline ENT vs CNT & $170-230$ & ENT & SPL & 7 & $\sim 80$ & $\begin{array}{c}\text { Only at the right } \\
\text { hemisphere }\end{array}$ \\
ET vs CT & $160-360$ & ET & IPL & $22,39-40$ & $\sim 170$ & $\begin{array}{c}\text { Only at the right } \\
\text { hemisphere }\end{array}$ \\
\hline
\end{tabular}

Abbreviations: CNT, control non-target; CT, control target; ENT, epileptic non-target; ET, epileptic target; IPL, inferior parietal lobule; SPL, superior parietal lobule.

In this time-range, the SPL was activated more in the ENT group; the activation of the SPL was mostly at the right hemisphere (Figure 1). The pair $t$ test between ET and CT revealed a statistically significant difference in the time-range of $160 \mathrm{~ms}$ to $360 \mathrm{~ms}$. In this time-range, the IPL was activated more in the ET group (only at the right hemisphere) (Figure 2).

The above findings are summarized in Table 5. 
Finally, regarding the comparison between methods, the statistical analysis revealed no significant differences between the three methods with respect to the voxels that were identified as statistically significant based on the contract analysis between the groups and tasks. In particular, there were no specific areas (over 50 voxels) with significant different activation for more than $50 \mathrm{~ms}$ duration. Of course, this may be due to the low number of EEG recordings used for the source-localization analysis. We discuss this issue next.

\section{4 | CONCLUSIONS}

Sensor-level analysis does not provide information about the actual sources that are involved in brain activity. From a mathematical point of view, the problem of source localization from scalp recordings is an inverse ill-defined problem and as such, different types of regularization approaches may in principle result to different solutions. Thus, especially for clinical assessment of brain neurological disorders such as epilepsy, there is a need for comparing and assessing the robustness of such methods.

This is the first study to perform a comparative analysis of three numerical methods, namely the sLORETA, wMNE and ASPM to identify differences at the source level between healthy children and children with well-controlled epilepsy (ie, in the absence of seizures) during a working memory task. In the absence of anatomical MRI scans, we used the publicly available "Neurodevelopmental MRI database" that provides age-specific average MRI templates.

In particular, we studied the localization of the sources of brain activity of children with BRE and idiopathic GE (including childhood absence epilepsy and GE with tonic-clonic seizures). These types of epilepsy are traditionally seen as distinct clinical entities: however, this difference appears to be much less pronounced. For example, Verrotti et al ${ }^{66,67}$ showed that BRE and childhood absence epilepsy may be pathophysiologically related, and the two epileptic phenotypes may indicate a neurobiological continuum. In another study, it was found that "children with BRE may have slightly lower verbal IQs than normal controls, but similar verbal deficiencies to children with idiopathic GE". ${ }^{68}$ Furthermore, as the interim analysis in the work of Myatchin et $\mathrm{al}^{7}$ showed, there were no statistically significant differences between those two epilepsy subgroups in terms of the brain activity recorded by EEG. Importantly, the source analysis that we conducted in the current work, showed that there were no statistically significant differences between the two types of epilepsy for the specific working memory task.

To avoid an acute effect of epileptic seizures on the child's performance, only patients with at least an 8 days seizure-free period preceding the test were included. Tromp et $\mathrm{al}^{69}$ found no significant decline in cognitive testing performance in children with frequent seizures ( $>1$ /week) comparing to children with sporadic seizures ( $<1 /$ week). In the study of Bailet and Turk $^{70}$ in children with epilepsy and normal IQ, no difference in performance on neurocognitive tests was found between patients with one or more (up to five) seizures in 1 month preceding the testing vs patients with no seizures in the last month. Therefore, 8 days of being seizure-free is a long enough period to avoid an acute effect of seizures on neurocognitive performance. Next, the majority of children in our study had much longer seizurefree period: median seizure-free period of 8 months with only two patients being seizure free for 3 weeks (for details see Myatchin et $\mathrm{al}^{7}$ ).

Our analysis shows that all three methods yield essentially the same results, thus providing adequate confidence for our findings. More specifically, our analysis revealed consistent differences between the two groups in the parietal lobes. Importantly, our findings are in line with other studies investigating abnormalities of the brain function due to epilepsy with the use of anatomical MRI, fMRI and EEG recordings. ${ }^{71-80}$ More specifically, regarding children with epilepsy, Besenyei et $\mathrm{al}^{80}$ used anatomical MRI and resting state EEG recordings to identify the abnormal brain activity in children with benign rolandic epilepsy. Using LORETA, they found an increase activity, compared to controls, in the temporal and inferior parietal lobule. Other studies that have investigated the abnormal activity in children with epilepsy in the presence of seizures have also pinpointed the importance of these areas. Clemens et $\mathrm{l}^{81}$ find increased activity in the superior parietal lobe children with benign childhood epilepsy with rolandic spikes, using MRI scans and resting state EEG recordings. For the source localization analysis they used LORETA.

Importantly, our study and findings reveal also the importance and potential that originates from the use of publicly available scientific resources such as the "Neurodevelopmental MRI" database, which allow to the researchers to reanalyze available neuroimaging data and investigate questions beyond the scope of the original studies. This carries, in principle, the potential to gain new insights without the need to perform new from scratch, time-consuming and expensive experiments. 
We are aware of the low number of participants that contained 21 epileptic children and a similar size of healthy children that served as a control group. However, other relevant studies have used even smaller number of subjects, yet leading to consistent results. ${ }^{24-26}$ Thus, our findings can serve as a basis for future studies that will consider a bigger number of subjects.

Finally, we would like to comment on the small number of electrodes that were used. The EEG signals have been recorded for the purposes of a clinical study using 19 electrodes with 4 additional EOG electrodes that may result to a blurred source localization in specific areas such as the temporal lobe. ${ }^{82}$ However, this does not mean that a low spatial sampling precludes source localization. For example, as it has been shown in a comparative study for epileptic sourcelocalization in children using EEGs recorded from 19 to 29 scalp electrodes, valuable insight about the underlying sources could be also extracted with the use of 19 electrodes. ${ }^{82}$ In our study, despite the small number of electrodes, the source analysis revealed differences between the two groups which are consistent with what has been found in other studies as discussed above.

\section{ACKNOWLEDGMENTS}

Evangelos Galaris was supported by a Ph.D. fellowship by the Department of Mathematics and Applications, University of Naples Federico II and Ioannis Gallos was supported by a Ph.D. fellowship by the National Technical University of Athens.

\section{CONFLICT OF INTEREST}

All authors declare no conflicts of interest in this paper.

\section{ORCID}

Ivan Myatchin (1) https://orcid.org/0000-0001-8857-3353

Constantinos Siettos ${ }^{-1}$ https://orcid.org/0000-0002-9568-3355

\section{REFERENCES}

1. Aaberg KM, Gunnes N, Bakken IJ, et al. Incidence and prevalence of childhood epilepsy: a nationwide cohort study. Pediatrics. 2017;139 (5):e20163908. https://doi.org/10.1542/peds.2016-3908.

2. Davies S, Heyman I, Goodman R. A population survey of mental health problems in children with epilepsy. Dev Med Child Neurol. 2003; 45(5):292-295. https://doi.org/10.1017/s0012162203000550.

3. Goodin DS, Aminoff J, Laxer K. Detection of epileptiform activity by different noninvasive EEG methods in complex partial epilepsy. Ann Neurol. 1990;27:330-334. https://doi.org/10.1002/ana.410270317.

4. Smith SJM. EEG in the diagnosis, classification, and management of patients with epilepsy. J Neurol Neurosurg Psychiatry. 2005;76(suppl 2):ii2-ii7. https://doi.org/10.1136/jnnp.2005.069245.

5. Jan MMS, Sadler M, Rahey SR. Lateralized postictal EEG delta predicts the side of seizure surgery in temporal lobe epilepsy. Epilepsia. 2001;42(3):402-405. https://doi.org/10.1046/j.1528-1157.2001.45999.x.

6. Krause CM, Boman PA, Sillanmäki L, Varho T, Holopainen IE. Brain oscillatory EEG event-related desynchronization (ERD) and -sychronization (ERS) responses during an auditory memory task are altered in children with epilepsy. Seizure. 2008;17(1):1-10. https:// doi.org/10.1016/j.seizure.2007.05.015.

7. Myatchin I, Mennes M, Wouters H, Stiers P, Lagae L. Working memory in children with epilepsy: an event-related potentials study. Epilepsy Res. 2009;86:183-190.

8. Protopapa F, Siettos CI, Evdokimidis I, Smyrnis N. Granger causality analysis reveals distinct spatio-temporal connectivity patterns in motor and perceptual visuo-spatial working memory. Front Comput Neurosci. 2014;8:146. https://doi.org/10.3389/fncom.2014.00146.

9. Yang L, Shklyar I, Lee HW, et al. Impaired consciousness in epilepsy investigated by a prospective responsiveness in epilepsy scale (RES). Epilepsia. 2012;53(3):437-447. https://doi.org/10.1111/j.1528-1167.2011.03341.x.

10. Diessen vE, Otte WM, Braun KPJ, Stam CJ, Jansen FE. Improved diagnosis in children with partial epilepsy using a multivariable prediction model based on EEG network characteristics. PLoS One. 2013;8(4):1-8. https://doi.org/10.1371/journal.pone.0059764.

11. Plummer C, Harvey AS, Cook M. EEG source localization in focal epilepsy: where are we now? Epilepsia. 2008;49(2):201-218. https:// doi.org/10.1111/j.1528-1167.2007.01381.x.

12. Mégevand P, Seeck M. Electroencephalography, magnetoencephalography and source localization. Curr Opin Neurol. 2018;31(2):176183. https://doi.org/10.1097/wco.0000000000000545.

13. Kaiboriboon K, Lüders HO, Hamaneh M, Turnbull J, Lhatoo SD. EEG source imaging in epilepsy-practicalities and pitfalls. Nat Rev Neurol. 2012;8(9):498-507. https://doi.org/10.1038/nrneurol.2012.150.

14. Ebersole JS, Ebersole SM. Combining MEG and EEG source modeling in epilepsy evaluations. J Clin Neurophysiol. 2010;27(6):360-371. https://doi.org/10.1097/wnp.0b013e318201ffc4. 
15. Moeller F, Stephani U, Siniatchkin M. Simultaneous EEG and fMRI recordings (EEG-fMRI) in children with epilepsy. Epilepsia. 2013;54 (6):971-982. https://doi.org/10.1111/epi.12197.

16. Jatoi MA, Kamel N, Malik AS, Faye I. EEG based brain source localization comparison of sLORETA and eLORETA. Australas Phys Eng Sci Med. 2014;37(4):713-721. https://doi.org/10.1007/s13246-014-0308-3.

17. Cincotti F, Babiloni C, Miniussi C, et al. EEG deblurring techniques in a clinical context. Methods Inf Med. 2004;43(1):114-117. https:// doi.org/10.1055/s-0038-1633846.

18. Yao J, Dewald JP. Evaluation of different cortical source localization methods using simulated and experimental EEG data. Neuroimage. 2005;25(2):369-382. https://doi.org/10.1016/j.neuroimage.2004.11.036.

19. Attal Y, Schwartz D. Assessment of subcortical source localization using deep brain activity imaging model with minimum norm operators: a MEG study. PLoS One. 2013;8(3):e59856. https://doi.org/10.1371/journal.pone.0059856.

20. Seeland A, Krell MM, Straube S, Kirchner EA. Empirical comparison of distributed source localization methods for single-trial detection of movement preparation. Front Hum Neurosci. 2018;12:340. https://doi.org/10.3389/fnhum.2018.00340.

21. Ioannides AA, Liu L, Poghosyan V, Hamandi K, Kostopoulos GK. (2015) Source-Estimation from Non-invasive Recordings of Brain Electrical Activity in Sleep and Epilepsy. In: Voros N., Antonopoulos C. (eds) Cyberphysical Systems for Epilepsy and Related Brain Disorders. Springer, Cham. https://doi.org/10.1007/978-3-319-20049-1_4

22. Chowdhury R, Merlet I, Birot G, et al. Complex patterns of spatially extended generators of epileptic activity: comparison of source localization methods cMEM and 4-ExSo-MUSIC on high resolution EEG and MEG data. Neuroimage. 2016;143:175-195. https://doi.org/10. 1016/j.neuroimage.2016.08.044.

23. Hassan M, Merlet I, Mheich A, et al. Identification of interictal epileptic networks from dense-EEG. Brain Topogr. 2016;30(1):60-76. https://doi.org/10.1007/s10548-016-0517-z.

24. Adebimpe A, Aarabi A, Bourel-Ponchel E, Mahmoudzadeh M, Wallois F. EEG resting state functional connectivity analysis in children with benign epilepsy with centrotemporal spikes. Front Neurosci. 2016;10:143. https://doi.org/10.3389/fnins.2016.00143.

25. Groening K, Brodbeck V, Moeller F, et al. Combination of EEG-fMRI and EEG source analysis improves interpretation of spikeassociated activation networks in paediatric pharmacoresistant focal epilepsies. Neuroimage. 2009;46(3):827-833. https://doi.org/10.1016/ j.neuroimage.2009.02.026.

26. Elshoff L, Groening K, Grouiller F, et al. The value of EEG-fMRI and EEG source analysis in the presurgical setup of children with refractory focal epilepsy. Epilepsia. 2012;53(9):1597-1606. https://doi.org/10.1111/j.1528-1167.2012.03587.x.

27. Fergus P, Hignett D, Hussain A, Al-Jumeily D, Abdel-Aziz K. Automatic epileptic seizure detection using scalp EEG and advanced artificial intelligence techniques. Biomed Res Int. 2015;2015:17. https://doi.org/10.1155/2015/986736.

28. Swanson HL. Cross-sectional and incremental changes in working memory and mathematical problem solving. J Educ Psychol. 2006;98 (2):265-281. https://doi.org/10.1037/0022-0663.98.2.265.

29. Gathercole SE, Pickering SJ, Knight C, Stegmann Z. Working memory skills and educational attainment: evidence from national curriculum assessments at 7 and 14 years of age. Appl Cogn Psychol. 2004;18(1):1-16. https://doi.org/10.1002/acp.934.

30. Gathercole SE, Alloway TP, Kirkwood HJ, Elliott JG, Holmes J, Hilton KA. Attentional and executive function behaviours in children with poor working memory. Learn Individ Differ. 2008;18(2):214-223. https://doi.org/10.1016/j.lindif.2007.10.003.

31. Toth JA, Lewis M. The role of working memory and external representation in individual decision making. AAAI Technical Report; 2003: 11.

32. Ruff CC, Knauff M, Fangmeier T, Spreer J. Reasoning and working memory: common and distinct neuronal processes. Neuropsychologia. 2003;41(9):1241-1253. https://doi.org/10.1016/S0028-3932(03)00016-2.

33. Gülgönen S, Demirbilek V, Korkmaz B, Dervent A, Townes BD. Neuropsychological functions in idiopathic occipital lobe epilepsy. Epilepsia. 2000;41(4):405-411. https://doi.org/10.1111/j.1528-1157.2000.tb00181.x.

34. Chaix Y, Laguitton V, Lauwers-Cancès V, et al. Reading abilities and cognitive functions of children with epilepsy: influence of epileptic syndrome. Brain Dev. 2006;28(2):122-130. https://doi.org/10.1016/j.braindev.2005.06.004.

35. Campo P, Maestú F, García-Morales I, et al. Modulation of medial temporal lobe activity in epilepsy patients with hippocampal sclerosis during verbal working memory. J Int Neuropsychol Soc. 2009;15:536-546. https://doi.org/10.1017/S135561770909078X.

36. Wagner DD, Sziklas V, Garver KE, Jones-Gotman M. Material-specific lateralization of working memory in the medial temporal lobe. Neuropsychologia. 2009;47(1):112-122. https://doi.org/10.1016/j.neuropsychologia.2008.08.010.

37. Luton LM, Burns TG, DeFilippis N. Frontal lobe epilepsy in children and adolescents: a preliminary neuropsychological assessment of executive function. Arch Clin Neuropsychol. 2010;25(8):762-770. https://doi.org/10.1093/arclin/acq066.

38. Pascual-Marqui RD. Standardized low-resolution brain electromagnetic tomography (sLORETA): technical details. Methods Find Exp Clin Pharmacol. 2002;24D:5-12.

39. Dale AM, Liu AK, Fischl BR, et al. Dynamic statistical parametric mapping: combining fMRI and MEG for high-resolution imaging of cortical activity. Neuron. 2000;26(1):55-67. https://doi.org/10.1016/S0896-6273(00)81138-1.

40. Iwaki S, Ueno S. Weighted minimum-norm source estimation of magnetoencephalography utilizing the temporal information of the measured data. J Appl Phys. 1998;83:6441-6443. https://doi.org/10.1063/1.367732.

41. Otsuka Y, Osaka N, Osaka M. Functional asymmetry of superior parietal lobule for working memory in the elderly. Neuroreport. 2008; 19:1355-1359. https://doi.org/10.1097/WNR.0b013e32830e000f.

42. Koenigs M, Barbey AK, Postle BR, Grafman J. Superior parietal cortex is critical for the manipulation of information in working memory. J Neurosci. 2009;29(47):14980-14986. https://doi.org/10.1523/JNEUROSCI.3706-09.2009. 
43. Olson IR, Berryhill M. Some surprising findings on the involvement of the parietal lobe in human memory. Neurobiol Learn Mem. 2009; 91(2):155-165. https://doi.org/10.1016/j.nlm.2008.09.006.

44. Ravizza S, Behrmann M, Fiez J. Right parietal contributions to verbal working memory: spatial or executive? Neuropsychologia. 2005;43: 2057-2067. https://doi.org/10.1016/j.neuropsychologia.2005.03.014.

45. Delorme A, Makeig S. EEGLAB: an open source toolbox for analysis of single-trial EEG dynamics including independent component analysis. J Neurosci Methods. 2004;134(1):9-21. https://doi.org/10.1016/j.jneumeth.2003.10.009.

46. Kybartaite A. Computational representation of a realistic head and brain volume conductor model: electroencephalography simulation and visualization study. Int J Numer Methods Biomed Eng. 2012;28(11):1144-1155. https://doi.org/10.1002/cnm.2483.

47. Fuchs M, Wagner M, Kastner J. Boundary element method volume conductor models for EEG source reconstruction. Clin Neurophysiol. 2001;112(8):1400-1407. https://doi.org/10.1016/S1388-2457(01)00589-2.

48. Wolters C. The finite element method in EEG/MEG source analysis. SIAM News. 2007;40.

49. Hallez H, Vanrumste B, Grech R, et al. Review on solving the forward problem in EEG source analysis. J Neuroeng Rehabil. 2007;4(1): 46. https://doi.org/10.1186/1743-0003-4-46.

50. Pascual-Marqui RD, Lirio RJ. Spatial resolution of neuronal generators based on EEG and MEG measurements. Int J Neurosci. 1993;68: 93-105. https://doi.org/10.3109/00207459308994264.

51. Hämäläinen MS, Ilmoniemi RJ. Interpreting magnetic fields of the brain: minimum norm estimates. Med Biol Eng Comput. 1994;32(1): 35-42. https://doi.org/10.1007/BF02512476.

52. Stenroos M, Hauk O. Minimum-norm cortical source estimation in layered head models is robust against skull conductivity error. Neuroimage. 2013;81:265-272. https://doi.org/10.1016/j.neuroimage.2013.04.086.

53. Grech R, Cassar T, Muscat J, et al. Review on solving the inverse problem in EEG source analysis. J Neuroeng Rehabil. 2008;5(1):25. https://doi.org/10.1186/1743-0003-5-25.

54. Backus G, Gilbert F. The resolving power of gross earth data. Geophys J Int. 1968;16(2):169-205. https://doi.org/10.1111/j.1365-246X. 1968.tb00216.x.

55. Richards JE, Sanchez C, Phillips-Meek M, Xie W. A database of age-appropriate average MRI templates. Neuroimage. 2016;124:12541259.

56. Evans AC. The NIH MRI study of normal brain development. Neuroimage. 2006;30(1):184-202. https://doi.org/10.1016/j.neuroimage. 2005.09.068.

57. Sanchez CE, Richards JE, Almli CR. Age-specific MRI templates for pediatric neuroimaging. Dev Neuropsychol. 2012;37(5):379-399. https://doi.org/10.1080/87565641.2012.688900.

58. Richards JE, Xie W. Brains for all the ages: structural neurodevelopment in infants and children from a life-span perspective. Adv Child Dev Behav. 2015;48:1-52.

59. Wendel-Mitoraj K, Väisänen J, Seemann G, Hyttinen J, Malmivuo J. The influence of age and skull conductivity on surface and subdermal bipolar EEG leads. Comput Intell Neurosci. 2010;2010:397272. https://doi.org/10.1155/2010/397272.

60. McCann H, Pisano G, Beltrachini L. Variation in reported human head tissue electrical conductivity values. Brain Topogr. 2019;32(5): 825-858. https://doi.org/10.1007/s10548-019-00710-2.

61. BenSaida A. Shapiro-Wilk and Shapiro-Francia normality tests. MATLAB Central File Exchange; 2019. https://www.mathworks.com/ matlabcentral/fileexchange/13964-shapiro-wilk-and-shapiro-francia-normality-tests. Accessed October 20, 2020.

62. Benjamini Y, Hochberg Y. Controlling the false discovery rate-a practical and powerful approach to multiple testing. J R Stat Soc Ser B. 1995;57:289-300. https://doi.org/10.2307/2346101.

63. Baillet S, Mosher J, Leahy R, Shattuck D. Brainstorm: a matlab toolbox for the processing of MEG and EEG signals. Neuroimage. 1999;9: S246.

64. Gramfort A, Papadopoulo T, Olivi E, Clerc M. OpenMEEG: opensource software for quasistatic bioelectromagnetics. Biomed Eng Online. 2010;9:45. https://doi.org/10.1186/1475-925X-9-45.

65. Gramfort A, Luessi M, Larson E, et al. MNE software for processing MEG and EEG data. Neuroimage. 2013;86:446-460. https://doi.org/ 10.1016/j.neuroimage.2013.10.027.

66. Verrotti A, D'Alonzo R, Rinaldi VE, Casciato S, D'Aniello A, Di Gennaro G. Childhood absence epilepsy and benign epilepsy with centro-temporal spikes: a narrative review analysis. World J Pediatr. 2017;13:106-111. https://doi.org/10.1007/s12519-017-0006-9.

67. Verrotti A, Casciato S, Spalice A, et al. Coexistence of childhood absence epilepsy and benign epilepsy with centrotemporal spikes: a case series. Eur J Paediatr Neurol. 2017;21(3):570-575. https://doi.org/10.1016/j.ejpn.2017.02.002.

68. Jeong MH, Yum MS, Ko TS, You SJ, Lee EH, Yoo HK. Neuropsychological status of children with newly diagnosed idiopathic childhood epilepsy. Brain Dev. 2011;33(8):666-671. https://doi.org/10.1016/j.braindev.2010.11.003.

69. Tromp SC, Weber JW, Aldenkamp AP, Arends J, Linden vI, Diepman L. Relative influence of epileptic seizures and of epilepsy syndrome on cognitive function. J Child Neurol. 2003;18(6):407-412. https://doi.org/10.1177/08830738030180060501.

70. Bailet LL, Turk WR. The impact of childhood epilepsy on neurocognitive and behavioral performance: a prospective longitudinal study. Epilepsia. 2000;41(4):426-431.

71. Berryhill ME, Olson IR. Is the posterior parietal lobe involved in working memory retrieval? Evidence from patients with bilateral parietal lobe damage. Neuropsychologia. 2008;46(7):1775-1786. https://doi.org/10.1016/j.neuropsychologia.2008.03.005.

72. Jones K, Berryhill M. Parietal contributions to visual working memory depend on task difficulty. Front Psych. 2012;3:81. https://doi.org/ 10.3389/fpsyt.2012.00081. 
73. Osaka M, Komori M, Morishita M, Osaka N. Neural bases of focusing attention in working memory: an fMRI study based on group differences. Cogn Affect Behav Neurosci. 2007;7:130-139. https://doi.org/10.3758/CABN.7.2.130.

74. Li S, Jin JN, Wang X, Qi HZ, Liu ZP, Yin T. Theta and alpha oscillations during the retention period of working memory by rTMS stimulating the parietal lobe. Front Behav Neurosci. 2017;11:170. https://doi.org/10.3389/fnbeh.2017.00170.

75. Tseng P, Iu KC, Juan CH. The critical role of phase difference in theta oscillation between bilateral parietal cortices for visuospatial working memory. Sci Rep. 2018;8(1):349. https://doi.org/10.1038/s41598-017-18449-w.

76. Hermann B, Jones J, Sheth R, Dow C, Koehn M, Seidenberg M. Children with new-onset epilepsy: neuropsychological status and brain structure. Brain. 2006;129(10):2609-2619. https://doi.org/10.1093/brain/awl196.

77. Hu CY, Gao X, Long L, et al. Altered DMN functional connectivity and regional homogeneity in partial epilepsy patients: a seventy cases study. Oncotarget. 2017;8(46):81475-81484. https://doi.org/10.18632/oncotarget.20575.

78. Kipp KH, Opitz B, Becker M, et al. Neural correlates of recognition memory in children with febrile seizures: evidence from functional magnetic resonance imaging. Front Hum Neurosci. 2012;6:17. https://doi.org/10.3389/fnhum.2012.00017.

79. Michels L, Bucher K, Lüchinger R, et al. Simultaneous EEG-fMRI during a working memory task: modulations in low and high frequency bands. PLoS One. 2010;5(4):1-15. https://doi.org/10.1371/journal.pone.0010298.

80. Besenyei M, Varga E, Fekete I, et al. EEG background activity is abnormal in the temporal and inferior parietal cortex in benign rolandic epilepsy of childhood: a LORETA study. Epilepsy Res. 2012;98(1):44-49. https://doi.org/10.1016/j.eplepsyres.2011.08.013.

81. Clemens B, Puskás S, Spisák T, et al. Increased resting-state EEG functional connectivity in benign childhood epilepsy with centrotemporal spikes. Seizure. 2016;35:50-55. https://doi.org/10.1016/j.seizure.2016.01.001.

82. Sperli F, Spinelli L, Seeck M, Kurian M, Michel CM, Lantz G. EEG source imaging in pediatric epilepsy surgery: a new perspective in presurgical workup. Epilepsia. 2006;47(6):981-990. https://doi.org/10.1111/j.1528-1167.2006.00550.x.

How to cite this article: Galaris E, Gallos I, Myatchin I, Lagae L, Siettos C. Electroencephalography source localization analysis in epileptic children during a visual working-memory task. Int J Numer Meth Biomed Engng. 2020;36:e3404. https://doi.org/10.1002/cnm.3404 